\title{
Edge Detection in Color Images using RGB Color Model
}

\author{
E. Boopathi Kumar \\ Research Scholar \\ PG \& Research Department of Computer Science \\ Gobi Arts \& Science College \\ Gobichettipalayam, Erode, India
}

\author{
V. Thiagarasu \\ Associate Professor \\ PG \& Research Department of Computer Science \\ Gobi Arts \& Science College \\ Gobichettipalayam, Erode, India
}

\begin{abstract}
Edge Detection is an important technique in image processing and it is the process of grouping an image into units that are consistent with respect to one or more features. Edge detection using gray images has lot of methods to segment and it has several set of algorithms to represent it. But the images produce more information in scenes i.e., color images have few set of methods to segment it. So, this paper represent color image edge detection methods in the literature and getting to prepare novel segmentation method by extracting the color channels in the RGB image into three with combined form of masking, filtering and Thresholding methods. Otsu method is one of the best and famous Thresholding method used in color image segmentation and it uses various combinations of masks to scan over the image to detect the correct boundary. Otsu method divides the segmentation tasks in two or more phases and provides the results better along with different phases. In the same way this paper discusses about RGB color model and fuzzy membership functions method and particularly about the usage of fuzzy membership functions which are used to create mask with some sort of rules based on RGB channel extraction to scan the separated channel image with few combinations and include Threshold method and filtering for further to produce the output image in well enhanced manner.
\end{abstract}

\section{Keywords}

Color Image Edge Detection, RGB Color Model, Color Channel Extraction, Fuzzy Inference System, Triangular Membership Function, Trapezoidal Membership Function.

\section{INTRODUCTION}

Segmentation is an image processing method, which divides the image into several regions with their own characteristic for the sake of extracting useful target, and it is a key step from the image processing to image analysis. It is one of the most important steps leading to the analysis of processed image data, which refers to grouping of similar pixels together and separating the particular portion of the image for the purpose of identification [1]. Its main goal is to divide an image into parts that have strong correlation with objects or areas of the real world contained in the image. Generally, Segmentation is the process of partitioning a digital image into multiple segments which gives more meaning and easier to analyze and cluster pixels into prominent image regions, i.e., regions corresponding to individual surfaces, objects, or natural parts of objects. Image segmentation algorithms are based on either discontinuity principle or similarity principle. The idea behind the discontinuity principle is to extract regions that differ in properties such as intensity, color, texture, or any other image statistics and the similarity principle is to group pixels based on common properties [2]. Basically Color is perceived by humans as a combination of tristimuli $R$ (red), $G$ (green), and $B$ (blue) which is usually called three primary colors [3]. From $R, G, B$ representation, we can derive other kinds of color model representations by using either linear or nonlinear type of transformations.

Basically, Color provides more powerful information for object detection in color images. In RGB image, each pixel has three color components: red, green, and blue. Amount of mixing of these three colors determines value of pixel. Image is a collection of pixels; each pixel is a combination of red, green, and blue colors for RGB image. So it is difficult to process each pixel; so, we need color model for robust detection. Here RGB color model is taken for research and process is carrying out through the same.

The paper is organized into five sections as follows: Section 2 discuss about the related works carried out in the field of color image Edge Detection. Section 3 discusses about the modules to be proposed for color channel extraction and edge detection based on Fuzzy logic Trapezoidal Membership Functions. Section 4 highlights discussion on the experiments to be done through Trapezoidal Membership Function by $2 \times 2$ masks and channel separation. Section 5 finally concludes the paper with future enhancement.

\section{RELATED WORK}

Firas Ajil Jassim proposed a novel algorithm based on combining two existing methods to obtain a significant method to partition the color image into significant regions. On their first phase, the traditional Otsu method for gray channel image segmentation were applied for each of the R,G, and $\mathrm{B}$ channels separately to determine the suitable automatic threshold for each channel. After that, the new modified channels are integrated again to formulate a new color image. The resulted image suffers from some kind of distortion. To get rid of those distortion, the second phase is arise which is the median filter to smooth the image and increase the segmented regions. Totally they used seven types of masks sizes to examine their work and conclude $15 \times 15$ produce clear results [4]. 
A.Kalaivani, Dr.S.Chitrakala represented K-Means Clustering algorithm which is the popular unsupervised clustering used for dividing the images into multiple regions based on image color property. The major issue of the algorithm is that the user has to specify the number of clusters- $\mathrm{K}$, which is used to split the image into $\mathrm{K}$ regions. To overcome the issue, they focused on determining $\mathrm{K}$ automatically based on local maxima of gray level co-occurrence matrix. Automatic generated $\mathrm{K}$ value is then passed to Fast $\mathrm{K}$-means Clustering algorithm for segmenting color images into multiple regions. They took RGB color model for their clustering process [5].

Navkirat Kaur presented color image segmentation algorithm in the form of color conversion. They convert RGB image to HSV because it gives the color according to human perception. Further three matrixes are made by three different planes. Firstly, a single new matrix is formed so as to see values of RGB at each pixel. If two rows are equal in a single new matrix then combine those rows. After that total number of colors existing in an original image is calculated. To see the exact color enter the number of colors wants to see and finally processed image is converted from HSV to RGB color space [7].

Rafael Guillermo Gonzalez Acuna generalizes Otsu's binarization method towards reduction of color levels in color images. Color defines a multi-dimensional property vector at each pixel location, and this can be further generalized towards considering arbitrarily finite-dimensional property vectors at pixel locations. Otsu's binarization method, originally already briefly discussed by Otsu for multiThresholding, was efficiently mapped earlier into a segmentation method for grey-level images by recursively applying the original binarization method. They generalize further by proposing a recursive algorithm for finite dimensional property vectors at pixel locations [8].

Suryakant, Neetu Kushwaha proposed the implementation of a very simple but efficient fuzzy logic based algorithm to detect the edges of an image without determining the threshold value. Their proposed approach begins by scanning the images using floating $3 \times 3$ pixel window. Fuzzy inference system is designed with 8 inputs, which corresponds to 8 pixels of instantaneous scanning matrix, one output that tells whether the pixel under consideration is "black", "white" or "edge" pixel. Rule base comprises of twenty eight rules, which classify the target pixel. The proposed method results for different captured images are compared to those obtained with the linear Sobel operator [9].

Ajaya Kumar, Banshidhar Majhi stated a new method of image segmentation by histogram thresholding based on the concept of fuzzy measure minimization. The membership function is used to express the unique association between a pixel and its belonging region which is either the object or the background. The optimal threshold can be effectively determined by minimizing the measure of fuzziness of the image. The main criteria for fuzzy based histogram threshoding approach are membership functions and fuzzy measures. While implementation, it has been noticed that bandwidth of s-membership function cannot be determined automatically. Other membership functions like Huang-wang, Gaussian, and Gamma are unable to produce same threshold irrespective of any fuzzy measure. Their membership function has provided consistent results to determine the global threshold in an image with respect to all described fuzzy measures [17].

Chaohui Lü, Xingyun Yang and Sha Qi implements a system of applying ant colony algorithm to image segmentation, which is based on the aspects of the discreteness of digital image and the fuzzy clustering ability of ant colony algorithm. There are mainly three contents in the algorithm. The first part is to extract the features of an image including the RGB values, the gradient, and the neighborhood. The second part is to set the clustering center with the method of a combination of statistics and artificial selection. And the third part is to apply the ant colony algorithm to segment a color image and they introduced a method based on the statistics and the artificial participation to find clustering centers [22].

Er. Manpreet Kaur, Ms. Sumeet Kaur represented a modified rule based fuzzy logic technique, because fuzzy logic is desirable to convert the uncertainties that exist in many aspects of image processing. Firstly the gradient and standard deviation is calculated and used as input for fuzzy system. The standard deviation and gradient values are used as input for fuzzy system using membership function .Fuzzy if-then else rules are applied to modify the membership to one of Low, medium and high classes. Finally defuzzification is performed. The three edge strength values used as fuzzy system inputs were fuzzified using Gaussian membership functions. Fuzzy if then rules are applied to modify the membership to one of low, medium, or high classes. The traditional algorithm like Sobel, Prewitt, LoG are implemented and then the results are compared with modified algorithm and concluded that the proposed technique is to find the more fine edges and reduce the pixels that are not belonging to the edge [27].

\section{MATERIALS \& METHODS}

Scanning the image by pixel is a challenging task to obtain the output. Masking is one of the important concepts which are used to scan the input image by pixel by pixel. It is an array based technique contains variables for each array attribute. In previous work, $2 * 2$ and $3 * 3$ masks are used based on different set of rule format. $2 * 2$ masks are in the form of 2 by 2 matrixes contains four input variables P1, P2, P3 and P4 which form a mask to scan the input image [10]. For $2 * 2$ masks, 16 rules are followed and $\mathrm{P} 4$ act as output variable. $3 * 3$ masks are in the form of 3 by 3 matrices having eight input variables such as P1, P2, P3, P4, P6, P7, P8 \& P9 and one output variable P5. Here P5 act as output variable which gives resultant pixel of the mask scan [11].

For $3 * 3$ masks, 28 rules were followed to scan the input image. Masks is slid over the whole image pixel by pixel row wise and the process continues till the time whole image is scanned for unwanted edge pixels. A Membership Function is 
a curve that defines how each point in the input space is mapped to a membership degree or value between 0 and 1 . Based on membership grade, input variables check the pixels of the image which is black, white or edge and output can be fetched with the base of mentioned fuzzy rules. Rules are feed in rule editor of fuzzy inference system engine which works on the concept of if else. User can add more number of variables which are suitable for problem of selection.

Likewise, this concept is taken further to process color image on the basis of color image segmentation. Here RGB color model is taken for research by extracting the image channels into three separate images. After separation all the three images are included for edge detection by using fuzzy membership functions. Following is an algorithm to perform extraction of the channels in given input image into three separate RGB channels.

\section{Color Separation Algorithm}

pic = imread('paint.jpg');

for $\mathrm{mm}=1: \operatorname{size}($ pic, 1$)$

for $\mathrm{nn}=1: \operatorname{size}($ pic, 2$)$

if $\operatorname{pic}(\mathrm{mm}, \mathrm{nn}, 1)<80 \& \& \operatorname{pic}(\mathrm{mm}, \mathrm{nn}, 2)>80 \& \& \operatorname{pic}(\mathrm{mm}$, $\mathrm{nn}, 3)>100$

$\mathrm{gsc}=0.3 * \operatorname{pic}(\mathrm{mm}, \mathrm{nn}, 1)+0.59 * \operatorname{pic}(\mathrm{mm}, \mathrm{nn}, 2)+0.11 *$ pic(mm, nn, 3);

$\operatorname{pic}(\mathrm{mm}, \mathrm{nn},:)=[\mathrm{gsc} g s c \mathrm{gsc}]$;

end

end

end

After extraction each image is taken further to include masking process through Mamdani inference system using membership functions. There are several kinds of membership functions present; they are Triangle, Trapezoidal, Gaussian, Bell, Sigmoid, Piecewise Linear, etc. Like grey process here too same membership (Triangle \& Trapezoidal) functions are planned to use.

\section{If-Then Rules}

Fuzzy sets and fuzzy operators are the subjects and verbs of fuzzy logic. These if-then rule statements are used to formulate the conditional statements that comprise the fuzzy logic. A single fuzzy if-then rule assumes the form if $\mathrm{x}$ is $\mathrm{A}$ then $\mathrm{y}$ is $\mathrm{B}$ where $\mathrm{A}$ and $\mathrm{B}$ are linguistic values defined by fuzzy sets on the ranges $\mathrm{X}$ and $\mathrm{Y}$, respectively. The if-part of the rule " $\mathrm{x}$ is $\mathrm{A}$ " is called the antecedent or premise, while the then-part of the rule " $y$ is $B$ " is called the consequent or conclusion. An example of such a rule might be

\section{If 'service' is 'good' then 'tip' is 'average'}

The concept good is represented as a number between 0 and 1 , and so the antecedent is an interpretation that returns a single number between 0 and 1 . Conversely, average is represented as a fuzzy set, and so the consequent is an assignment that assigns the entire fuzzy set $\mathrm{B}$ to the output variable $\mathrm{y}$. Likewise, for grey level images 0 and 1 denotes black and white in the form of pixel values. Edge can be found out through whether the pixel is black or white. To detect edges several set of rules are framed and implemented through inference systems' rule editor. Rules can be varied based on size of the masks. Two different set of masks are developed to scan the image with the implementation of two different set of rules format.

Table 1: Rule Table For 3 x 3 Mask

\begin{tabular}{|c|c|c|c|c|c|c|c|c|}
\hline \multicolumn{8}{|c|}{ Fuzzy Input } & \multirow{2}{*}{\begin{tabular}{|r|}
$\begin{array}{l}\text { Fuzzy } \\
\text { Output }\end{array}$ \\
P5
\end{tabular}} \\
\hline Pl & $\mathrm{P} 2$ & P3 & $\mathrm{P} 4$ & P6 & P7 & P8 & P9 & \\
\hline $\mathrm{W}$ & W & W & W & W & $B$ & $B$ & $B$ & $E$ \\
\hline B & B & $B$ & $\mathrm{~W}$ & W & $\mathrm{W}$ & $\mathrm{W}$ & $\mathrm{W}$ & $\bar{E}$ \\
\hline $\mathrm{B}$ & W & W & $B$ & W & $B$ & W & W & $E$ \\
\hline W & W & $\bar{B}$ & W & B & W & W & B & $E$ \\
\hline B & B & W & B & W & B & W & W & $\mathrm{E}$ \\
\hline W & W & $\bar{B}$ & W & B & W & B & $\bar{B}$ & $\mathrm{E}$ \\
\hline $\mathrm{B}$ & W & W & $B$ & W & $B$ & $B$ & W & $E$ \\
\hline W & $B$ & $\bar{B}$ & W & B & W & W & B & $E$ \\
\hline B & B & $\bar{B}$ & $\bar{B}$ & W & W & W & W & $\bar{E}$ \\
\hline $\bar{W}$ & W & $\bar{W}$ & $\bar{B}$ & W & B & B & $\bar{B}$ & $E$ \\
\hline $\mathrm{B}$ & $\mathrm{B}$ & $B$ & W & $B$ & $\mathrm{~W}$ & $\mathrm{~W}$ & W & $E$ \\
\hline W & W & W & W & $B$ & $B$ & $B$ & B & $E$ \\
\hline B & B & B & B & B & W & W & W & $E$ \\
\hline B & B & W & $\bar{B}$ & W & B & B & W & $\mathrm{E}$ \\
\hline $\mathrm{W}$ & W & W & B & B & B & $\bar{B}$ & B & $\mathrm{E}$ \\
\hline $\mathrm{W}$ & B & $\bar{B}$ & W & B & W & $\bar{B}$ & B & $\mathrm{E}$ \\
\hline B & W & W & B & W & W & W & W & $E$ \\
\hline W & W & W & B & W & B & W & $\bar{W}$ & $\mathrm{E}$ \\
\hline $\mathrm{W}$ & W & $\mathrm{W}$ & W & $\mathrm{W}$ & $B$ & $B$ & $\mathrm{~W}$ & $E$ \\
\hline B & $B$ & $\bar{W}$ & W & W & W & W & $\bar{W}$ & $\mathrm{E}$ \\
\hline $\mathrm{W}$ & W & W & W & W & $\mathrm{W}$ & $\bar{B}$ & B & $\mathrm{E}$ \\
\hline $\mathrm{W}$ & W & W & W & $B$ & $W$ & W & B & $E$ \\
\hline $\mathrm{W}$ & W & B & $\mathrm{W}$ & B & $\mathrm{W}$ & W & W & $E$ \\
\hline $\bar{W}$ & B & B & $\mathrm{W}$ & $\mathrm{W}$ & $\mathrm{W}$ & $\mathrm{W}$ & W & $\bar{E}$ \\
\hline $\mathrm{W}$ & B & B & W & B & W & $\mathrm{W}$ & $\mathrm{W}$ & $E$ \\
\hline W & W & W & W & B & W & B & B & $\mathrm{E}$ \\
\hline $\mathrm{B}$ & B & $\mathrm{W}$ & $B$ & W & W & W & $\mathrm{W}$ & $E$ \\
\hline W & W & W & B & W & B & B & W & $\mathrm{E}$ \\
\hline
\end{tabular}

\section{RESULTS \& DISCUSSION}

The results of membership functions such as trapezoidal and triangular methods used in previous process are shown in this section. Picture quality measures such as PSNR and MSE are used to compare the results of both membership functions. Results of triangular membership functions $2 * 2$ and $3 * 3$ masks are shown in the following figure 2 . Results of trapezoidal membership functions $2 * 2$ and $3 * 3$ masks are shown in the figure 1.

Here two different trapezoidal masks are used separately and their performances are measured. Among them Trapezoidal $3 * 3$ masks produces low error rate and high percentage of accuracy when compared to the methods using $3 * 3$ and $2 * 2$ of Triangular membership function. In existing work, edge detection have been done by fuzzy's inference system's triangular membership functions. In that, $2 * 2$ and $3 * 3$ mask are examined with the help of various fuzzy inference rules. 
Different input and output fuzzy sets are declared. In present works, fuzzy's trapezoidal membership function is used and $2 * 2[14]$ and $3 * 3$ [15] masks are generated. The result of both masks are compared to previous works and find that proposed work works well by finding exact edges in the given input image. The analysis can be done through finding edge plot percentage. For calculating the error rate in the proposed work, Mean Square Error and PSNR values are calculated using their formulas.

Following figures 1 and 2 shows the results of Triangular and Trapezoidal membership functions applied on grey level images in the combination of $2 \times 2$ and $3 \times 3$ masks. In that color image only taken as input image and then it will be converted to grey image. Inference system has five different functions such as Rule editor, Membership Function (MF) Editor, Fuzzy Inference System (FIS) Editor, Rule Viewer and Surface Viewer. In that Rule viewer and Surface Viewer are read only tools. Here, Converted image can be proceeding further into fuzzy inference system by using Rule editor, Membership function editor and Fuzzy Inference System Editor.
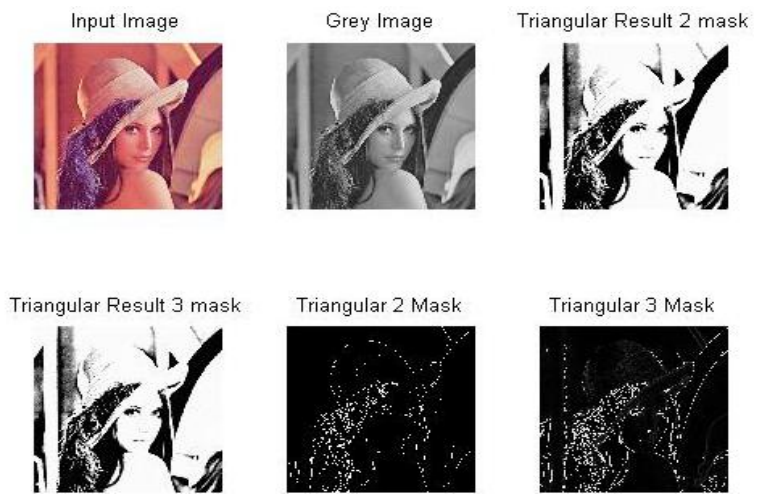

Figure 1: Triangular Membership Functions Results

Above figure describes the process carried out to find the edges in the image using triangular membership function. Here two different masks types are examined on grey level image. Firstly, input image is chosen and it will convert into grey image. After conversion input image is carried out to inference system to find the edges in image with the help of membership functions. This figure is an example of triangular membership function which is chosen for find edges. In the same way figure 2 shows the results of edge detected image which is carried out through trapezoidal membership function.

Trapezoidal result of $3 \times 3$ mask produce effective results when compared to all the other three mask including trapezoidal. It can be obtain by using picture quality measures as parameters. Color images express more information while compared to gray scale images. Basically, Color image segmentation follows discontinuity principles to extract the regions based on color as its property. There is large number of color image segmentation techniques based on segment properties which are demonstrated earlier. Segmentation properties can be classified into four general categories such as pixel-based, edge-based, region-based, and model-based techniques.
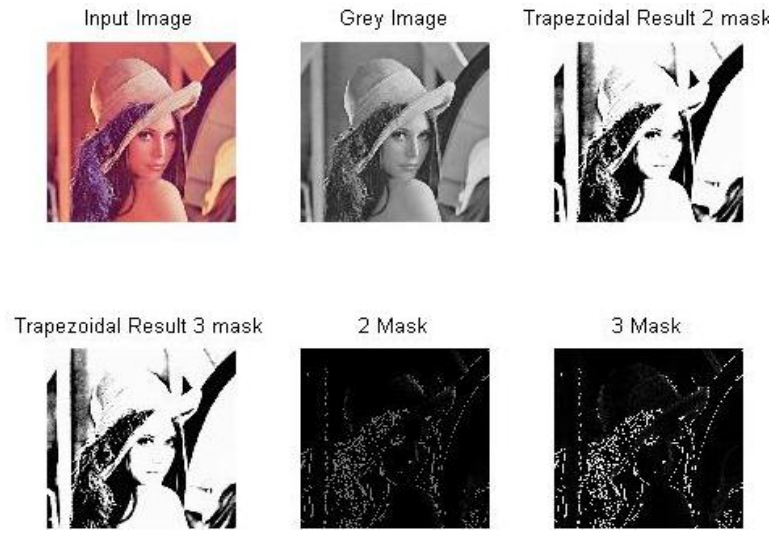

Figure 2: Trapezoidal Membership Functions Results

Actually, the basic behavior of these techniques can be divided into three major concepts. The first concept is the similarity concept which is like edge-based techniques which involves edge detection in image. Alternatively, the second concept is based on the discontinuity of pixel values as same as pixel-based and region-based techniques. It is an effective concept which is accepted overall by all categories of applications in literature. Finally, a complete different approach is the third concept which is based on a statistical approach like Model-based techniques. This technique provides approximate mathematical calculation in order of statistical way. There are various color models present such as RGB, CMY, and HSV etc, which are considered to examine color segmentation process. In this research work RGB color model is taken for execution.

Below figure is an example of color channel extraction which is used to extract the color channels from the image. Here RGB color model is chosen, so, three channels such as red, green and blue channels are extracted separately and all of them are included for edge detection technique to detect the edges present.

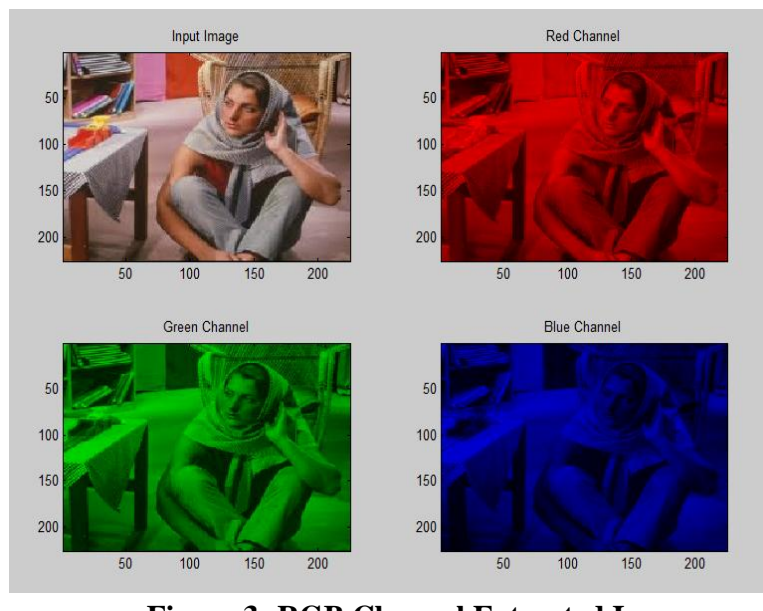

Figure 3: RGB Channel Extracted Image

All the three extracted images are included for edge detection technique by means of membership functions. Likewise grey images same procedure will be followed for these extracted images. Here scanning concept of pixel identification are done through $2 \times 2$ mask of triangular membership function. Same 
kind of rules is implemented through the rule editor. 16 set of rules which is used to identify the resultant pixel can be fixed as either black or RGB.

Table 2: Results of PSNR

\begin{tabular}{|cccccc|}
\hline PSNR & LENA & VEG & FLAG & PAINT & HAT \\
\hline SOBEL & 52.582 & 54.485 & 56.531 & 55.324 & 52.017 \\
\hline PREWIT & 52.351 & 53.261 & 54.266 & 53.587 & 51.754 \\
\hline TRI 3 M & 52.208 & 54.893 & 56.373 & 55.212 & 52.079 \\
\hline $\begin{array}{c}\text { TRAP 3 } \\
\text { M }\end{array}$ & 53.912 & 55.044 & 56.081 & 55.596 & 52.207 \\
\hline
\end{tabular}

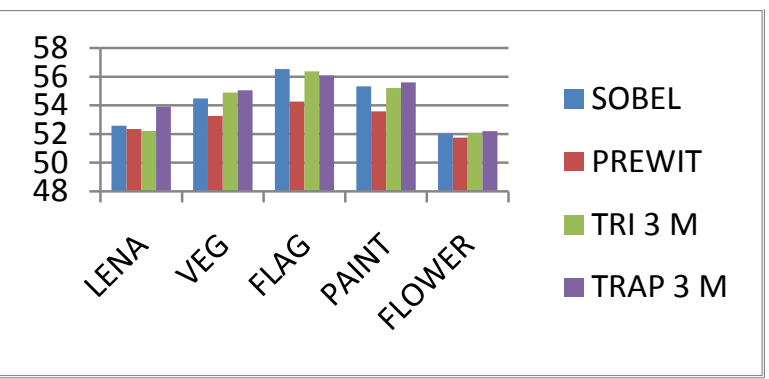

\section{Chart 1: Values of PSNR}

Edge percentages are calculated with the help of picture quality measures to show which method outperforms well among them. Peak signal-to-noise ratio, often abbreviated PSNR, is used to measure the image quality in this work. Table 2 shows the results obtained in edge detection on grey level process by four methods with five different image sets. Chart 1 displays the result range of PSNR with different image sets. The qualitative results show that Trapezoidal membership function produces high edge rate when compared to existing methods and this method has the advantage of keeping the more edge details than the other ways. Because the algorithm is an improved method of pixel processing using Membership functions based on three components of RGB color model and it compose the component edge to color image edge.

\section{CONCLUSION}

Edge Detection techniques used in image segmentation especially on color image has been represented in this paper. Each technique described in this work has its own advantage and disadvantage based on their segmentation properties. Many authors stated that combining two or more methods will produce effective segmentation results while applying to color images. On calculating the performance analysis, masking methods produce much better results with accuracy while comparing to other techniques in the literature. Variation in masking properties gives different types of results with respect to RGB color model and results proves that increasing window sizes makes images more clear. This paper concludes that color image segmentation using Trapezoidal membership function with the mask range of $2 \times 2$ and $3 \times 3$ produce better results in starting level window sizes by using Fuzzy rule based Inference system. Generally, implementation process can be done through in two entirely different phases. On the first phase, the fuzzy membership function based edge detection for gray channel image segmentation were applied for each of the R,G, and B channels separately to determine the suitable automatic threshold for each channel. After that, the new modified channels are included with channel wise and again to form a resultant color image. The resultant image suffers from some kind of alteration. To get rid of this warp, the second phase is arise which is the median filter to smooth the image and increase the segmented regions. Experimental results were presented on a variety of test images to support the proposed algorithm effectively.

\section{REFERENCES}

[1] Anil K Jain (2014), "Fundamentals of Digital Image Processing”, ISBN 978-81-203-0929-6, Pearson Education.

[2] Rafael C Gonzalez and Richard E Woods (2013), "Digital image processing", ISBN 978-81-317-2695-2, Pearson Education.

[3] Rafael C Gonzalez, Richard E Woods and Steven L Eddins (2011), "Digital image processing using MATLAB”, ISBN - 13: 978-0-07-070262-2, Tata McGraw Hill Education.

[4] Firas Ajil Jassim, Fawzi H. Altaani, "Hybridization of Otsu Method and Median Filter for Color Image Segmentation", International Journal of Soft Computing and Engineering (IJSCE) ISSN: 2231-2307, Volume-3, Issue-2, May 2013

[5] A.Kalaivani, Dr.S.Chitrakala, "Automatic Color Image Segmentation", International Conference on Science, Engineering and Management Research (ICSEMR 2014), (C)2014 IEEE.

[6] Md. Habibur Rahman, Md. Rafiqul Islam, "Segmentation of Color Image using Adaptive Thresholding and Masking with Watershed Algorithm", 978-1-4799-04006/13/ @2013 IEEE.

[7] Navkirat Kaur, V. K. Banga, Avneet Kaur, "Image Segmentation Based on Color", International Journal of Research in Engineering and Technology, Volume: 02, Issue: 11, Nov-2013

[8] Rafael Guillermo Gonzalez, Junli Tao, "Generalization of Otsu's Binarization into Recursive Color Image Segmentation”, 978-1-5090-0357-0/15/, (c) 2015 IEEE.

[9] Suryakant, Neetu Kushwaha, "Edge Detection using Fuzzy Logic in Matlab", International Journal of Advanced Research in Computer Science and Software Engineering, Volume 2, Issue 4, April 2012.

[10] Shikha Bharti, Sanjeev Kumar, "An Edge Detection Algorithm based on Fuzzy Logic", International Journal of Engineering Trends and Technology, Volume 4, Issue 3, 2013 .

[11] Mehul Thakkar, Prof. Hitesh Shah, "Edge Detection Techniques Using Fuzzy Thresholding", 978-1-46730126-8/ 2011, IEEE. 
[12] Song Gao, Chengcui Zhang, and Wei-Bang Chen, "An Improvement of Color Image Segmentation through Projective Clustering", IEEE IRI 2012, August 8-10, 2012. Jan Puzicha and Serge Belongie, "Model-based Halftoning for Color Image Segmentation", UC Berkeley, Department of Computer Science, December 2002.

[13] Soumya Dutta, Bidyut B. Chaudhuri, "Homogenous Region based Color Image Segmentation", Proceedings of the World Congress on Engineering and Computer Science, ISBN: 978-988-18210-2-7, Volume 2, October 2009.

[14] E. Boopathi Kumar, M. Sundaresan, "Edge Detection Using Trapezoidal Membership Function Based on Fuzzy's Mamdani Inference System”, IEEE, 2014.

[15] E. Boopathi Kumar, M. Sundaresan, "Fuzzy Inference System based Edge Detection using Fuzzy Membership Functions", International Journal of Computer Applications, ISSN: 0975 - 8887, Volume 112, Issue: 4, February 2015

[16] Xiaohua Tian, Wang sheng Yu, "Color Image Segmentation Based on Watershed Transform and Feature Clustering", IEEE 2016.

[17] Ajaya Kumar Dash, Banshidhar Majhi, "Image Segmentation Using Fuzzy Based Histogram Thresholding", IEEE 2015.

[18] Sneha M. Mahajan, Yogita K. Dubey, "Color Image Segmentation Using Kernalized Fuzzy C-means Clustering", Fifth International Conference on Communication Systems and Network Technologies, IEEE 2015

[19] E. Boopathi Kumar, V. Thiagarasu, "Segmentation using Fuzzy Membership Functions: An Approach", IJCSE, ISSN 2347-2693, Pages: 101-105, Volume 5, Issue 3, March 2017

[20] E. Boopathi Kumar, V. Thiagarasu, "Segmentation using Fuzzy Logic in Color Images Based on Membership Functions", IJESRT, ISSN 2277 - 9655, Pages: 38-45, Volume 6, Issue 6, June 2017.

[21] Huang-Chia Shih, En-Rui Liu, "Automatic Reference Color Selection for Adaptive Mathematical Morphology and Application in Image Segmentation", IEEE Transactions On Image Processing, 2016.

[22] Chaohui Lü, Xingyun Yang and Sha Qi, "Color Image Segmentation Based on the Ant Colony Algorithm", th International Congress on Image and Signal Processing, IEEE, 2015 .

[23] Simranjit Singh Walia, Gagandeep Singh, "Color based Edge detection techniques- A review", International Journal of Engineering and Innovative Technology, Volume 3, Issue 9, March 2014

[24] E. Boopathi Kumar, V. Thiagarasu, "Segmentation using Masking Methods in Color Images: an Approach", International Journal of Engineering Sciences \& Research Technology (IJESRT), ISSN 2277 - 9655, Pages: 104-110, Volume 6, Issue 2, February 2017.

[25] E. Boopathi Kumar, V. Thiagarasu, "Comparison and Evaluation of Edge Detection using Fuzzy Membership Functions", International Journal on Future Revolution in Computer Science \& Communication Engineering (IJFRCSCE), ISSN: 2454 - 4248, Pages: 149 - 153, Volume 3, Issue 8, August 2017.

[26] Er. Manpreet Kaur, Ms. Sumeet Kaur, "A New Approach To Edge Detection Using Rule Based Fuzzy Logic", Volume 2, No. 9, September 2011.

[27] Emmanuel Joy and J. Dinesh Peter, "Tracking of Unique Colored Objects: A Simple, Fast Visual Object Detection and Tracking Technique", E.B. Rajsingh et al. (eds.), Informatics and Communication Technologies for Societal Development, Springer India 2015.

[28] M. Borsotti, P. Campadelli, R. Schettini, "Quantitative evaluation of color image segmentation results", Pattern Recognition Letters 19 (1998) 741-747

[29] H.D. Cheng, X.H. Jiang, Y. Sun, Jingli Wang, "Color image segmentation: advances and prospects", Pattern Recognition 34 (2001) 2259-2281.

[30] E. Boopathi Kumar, V. Thiagarasu, "Color Image Edge Detection Using Fuzzy Membership Functions", International Journal of Scientific Research in Science, Engineering and Technology (IJSRSET), ISSN: 23944099, Pages: 970 - 975, Volume 3, Issue 6, September October 2017. 